\title{
Snare technique for the remodeling of the redundant arachnoid pouch to prevent cerebrospinal fluid rhinorrhea and hematoma collection during transsphenoidal surgery for suprasellar-extended pituitary tumors
}

\author{
Ju Hyung Moon, MD, Eui Hyun Kim, MD, PhD, and Sun Ho Kim, MD, PhD \\ Department of Neurosurgery, Pituitary Tumor Center, Yonsei Endocrine Research Institute, Yonsei University College of \\ Medicine, Seoul, Korea
}

\begin{abstract}
OBJECTIVE Transsphenoidal surgery (TSS) is considered a most effective treatment for pituitary tumors with huge suprasellar extension. However, the chance of developing CSF leakage is relatively high, because tearing of the arachnoid membrane is common and there could be multiple tear points during the dissection of suprasellar tumors from the overlying arachnoid membrane. If there are multiple leaking points in the arachnoid membrane packing methods such as using fat or multilayered fascia graft may not be sufficient to seal off the leaking points. Moreover, the packing material may not provide sufficient tamponade to stop bleeding, and thus generates postoperative hematoma formation in the tumor resection cavity. To prevent these complications, the authors have developed a new technique for remodeling the redundant arachnoid pouch (the so-called snare technique) to reconstruct the diaphragm, seal off the CSF leak points completely, and reduce the dead space in the tumor resection cavity.
\end{abstract}

METHODS In 9 patients with huge macroadenomas (> $2.5 \mathrm{~cm}$ in diameter) with suprasellar extension, the snare technique was used to remodel the arachnoid pouch after tumor removal via standard TSS between July 2009 and August 2014. Complications were investigated, including postoperative CSF rhinorrhea, postoperative hematoma collection, and visual compromise.

RESULTS During the resection of the tumor, CSF leakage was encountered in 8 cases, all of which were sealed off using the snare technique. In 1 case without intraoperative CSF leakage, the snare technique was also applied after intentional puncturing of the arachnoid membrane to reduce the volume and tension of the arachnoid pouch. None of the 9 patients experienced postoperative CSF rhinorrhea. Lumbar CSF drainage was not required in any case. Magnetic resonance imaging studies performed 24 hours after surgery revealed a remarkable reduction in the height of the diaphragm in all cases. Visual deficits improved in all patients immediately after surgery.

CONCLUSIONS Remodeling of the arachnoid pouch using the snare technique is simple and effective for completely sealing off the CSF leak point and preventing hematoma collection in the tumor resection cavity after TSS for huge pituitary tumors with suprasellar extension.

http://thejns.org/doi/abs/10.3171/2015.11.JNS151328

KEY WORDS cerebrospinal fluid leakage; postoperative hematoma; snare technique; transsphenoidal surgery; pituitary surgery

$\mathrm{T}$ RANSSPHENOIDAL surgery (TSS) is now accepted as a standard treatment for most pituitary adenomas, offering effectiveness and safety. ${ }^{10,16,26}$ In cases of nonfunctioning pituitary adenomas, because of their endocrinologically silent nature, the diagnosis is frequently established when the tumor has grown to a large size, with eminent suprasellar extension causing visual symptoms. ${ }^{28,34}$ With advances in TSS, its excellent postopera- tive results have proven that this method is enormously effective for the treatment not only of intrasellar adenomas but also of huge adenomas with suprasellar extensions. ${ }^{6,13}$ Currently, TSS is recommended as the procedure of choice for most pituitary adenomas with suprasellar extension, with the exception of rare cases with an irregular multilobular shape and extremely marked parasellar extensions. ${ }^{14,27,33}$ 
Despite the safety of TSS, complications such as CSF rhinorrhea, diabetes insipidus, meningitis, visual worsening, oculomotor nerve palsy, hypothalamic injury, carotid artery injury, and oro-rhino-sinusal complications are always possible. ${ }^{3,5,7,9}$ The risk of CSF leakage is increased in the presence of a suprasellar extension of a tumor., ${ }^{5,89}$ Because the surface area of the tumor contacting the arachnoid membrane is relatively large in cases with huge suprasellar extension, the possibility of arachnoid tears during dissection of the tumor from the overlying arachnoid membrane is higher, the leak point of the arachnoid could be larger, and there could be multiple tear points. This type of CSF leakage is difficult to seal off with conventional repair techniques. CSF leakage may lead to more serious postoperative complications, such as meningitis and pneumocephalus, with fatal consequences. ${ }^{9,15,32}$ Moreover, a hematoma can occasionally accumulate in the large dead space that is created after the removal of huge suprasellar-extended macroadenomas (Fig. 1). This hematoma may push the redundant arachnoid membrane upward, causing visual impairment, hydrocephalus, lethargy, disturbance of consciousness, paresis, or even death. $5,9,11,15,21,24,32$ According to the literature, most postoperative sellar hematomas that require immediate reoperation occur in huge pituitary adenomas with suprasellar extension. . $^{1,4,711,29}$ For the prevention of these complications, we have developed a new technique for the reconstruction of the arachnoid membrane that have evaginated into the intrasellar space. In this report, we describe our technique and present our experiences in 9 patients.

\section{Methods \\ Clinical Applications}

Between July 2009 and August 2014, 798 patients underwent TSS performed by the senior neurosurgeon (S.H.K.) at Severance Hospital, Yonsei University College of Medicine, Seoul, Korea. Among these patients, we used the snare technique in 9 patients with huge macroadenomas ( $>2.5 \mathrm{~cm}$ in diameter) that extended into the suprasellar area (Table 1). There were 5 men and 4 women, whose ages ranged from 20 to 65 years (mean 48.2 years). Immunohistochemical analysis of tumor tissues showed negative staining for any pituitary hormones in all patients. According to the modified Hardy classification scheme, ${ }^{19,23}$ all patients were classified as Type III $(n=5)$ or Type IV ( $=4$ ). All patients showed visual symptoms before surgery. Standard conventional TSS was selected for the removal of the tumor in all patients. None of the patients had previously undergone transcranial surgery and/or TSS.

\section{Surgical Techniques \\ Approach to the Tumor}

All operative procedures were performed using a routine TSS technique. Intraoperative MRI was performed using a 0.15-T PoleStar N-20 system (Medtronic Navigation) in 8 cases, and electromagnetic navigation (Stealth Station AxiEM navigation system, Medtronic) was used in 1 case. A direct endonasal approach using the operative microscope was used for all patients, and endoscopic assistance was adopted in 2 cases for the exploration of
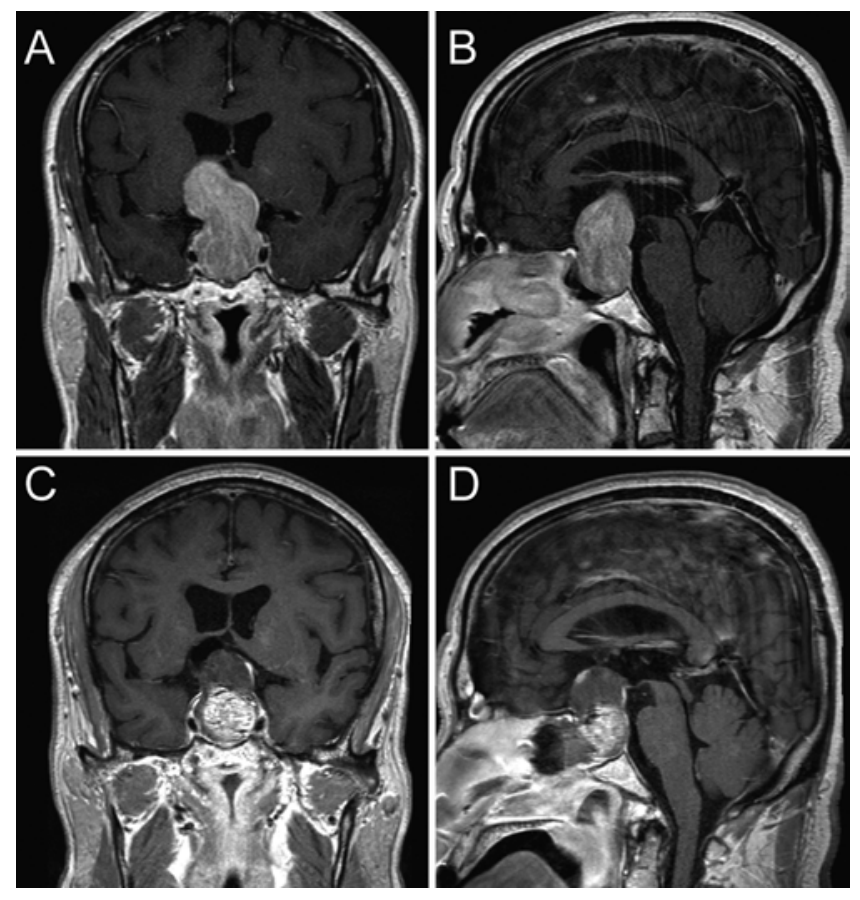

FIG. 1. MR images of a 65 -year-old male patient with postoperative suprasellar hematoma. A and B: Preoperative coronal and sagittal T1-weighted scans after Gd administration demonstrating a nonfunctioning macroadenoma with suprasellar extension. C and D: Postoperative coronal and sagittal T1-weighted scans after Gd administration showing a suprasellar hematoma that occupied the empty space created after tumor removal.

a hidden area that was invisible on a microscopic view. The superior turbinate was pushed laterally and an anterior sphenoidotomy was performed using a standard technique. Both carotid prominences were identified at the lower lateral margin of the surgical field. A bone window was made on the sellar floor to expose the medial boundaries of both cavernous sinuses. A rectangular dural incision was made to expose the margins of both medial walls of the cavernous sinus bilaterally. The location of the upper margin of the dural incision was determined according to the extent of the tumor over the diaphragm. Occasional minimal bleeding from the edges of the intercavernous sinus was controlled using a collagen fleece coated with a fibrin sealant patch (TachoSil; Nycomed).

\section{Tumor Removal and Snare Technique for Remodeling of the Redundant Arachnoid Pouch}

First, the intrasellar portion of the tumor was resected completely, starting from the floor side and extending to both sides. Subsequently, decompression of the suprasellar portion of the tumor was initiated. Necrotic tumor tissues were carefully resected using suction, ring curettes, and pituitary microcup forceps. When adhesion between the tough tumor capsule and the overlying arachnoid membrane or the normal gland was encountered, the capsule was carefully peeled off from the overlying arachnoid and the normal gland using a small piece of cellulose fabric (Bemsheet; Kawamoto Bandage Material Co.). This technique was described in detail in our previous publication. ${ }^{18}$ 
TABLE 1. Characteristics of the 9 patients who underwent the snare technique for the treatment of suprasellar tumors*

\begin{tabular}{|c|c|c|c|c|c|c|c|c|}
\hline \multirow{2}{*}{$\begin{array}{l}\text { Case } \\
\text { No. }\end{array}$} & \multirow{2}{*}{$\begin{array}{l}\text { Age (yrs), } \\
\text { Sex }\end{array}$} & \multirow{2}{*}{$\begin{array}{l}\text { Hardy } \\
\text { Type }\end{array}$} & \multirow{2}{*}{$\begin{array}{c}\text { Intraop Arachnoid } \\
\text { Tear }\end{array}$} & \multicolumn{3}{|c|}{$\begin{array}{l}\text { Suprasellar Extension Height Above } \\
\text { Diaphragm }(\mathrm{mm}) \dagger\end{array}$} & \multicolumn{2}{|c|}{ Visual Status } \\
\hline & & & & Preop & POD 1 & Postop 1 Yr & Preop & Postop \\
\hline 1 & $57, \mathrm{M}$ & IV & Yes, single hole & 26.9 & 13.4 & -8.6 & BTH & Improved \\
\hline 2 & $59, \mathrm{~F}$ & IV & Yes, multiple holes & 25.3 & 11.2 & -6.2 & BTH & Improved \\
\hline 3 & $51, \mathrm{~F}$ & IV & Yes, multiple holes & 18.0 & 9.8 & -4.7 & $\begin{array}{l}\text { OS superior quadrant } \\
\text { anopsia }\end{array}$ & Improved \\
\hline 4 & $64, \mathrm{~F}$ & III & Yes, single hole & 19.0 & 5.5 & -9.8 & OS hemianopsia & Improved \\
\hline 5 & $34, \mathrm{M}$ & IV & Yes, single hole & 25.6 & 14.5 & -3.1 & BTH & Improved \\
\hline 6 & $32, F$ & III & Yes, multiple holes & 19.4 & 6.3 & -1.2 & BTH & Improved \\
\hline 7 & $20, M$ & III & No & 20.3 & 12.5 & -3.7 & BTH & Improved \\
\hline 8 & $65, \mathrm{M}$ & III & Yes, multiple holes & 13.9 & 10.2 & Not yet completed & Peripheral dimness & Improved \\
\hline 9 & $52, \mathrm{M}$ & III & Yes, multiple holes & 44.3 & 13.9 & Not yet completed & BTH & Improved \\
\hline
\end{tabular}

After the complete removal of the intrasellar and suprasellar portions of the tumor, the arachnoid membrane and the normal gland, which had previously been located on the upper dome of the tumor, were evaginated into the intrasellar space. In conventional TSS, this evaginated arachnoid membrane is usually pushed up by intrasellar packing using pieces of autologous fat or muscle to prevent postoperative CSF rhinorrhea and achieve hemostasis. However, the fat or muscle packing may not provide sufficient tamponade to seal the CSF leak point completely and stop the bleeding due to redundancy of the arachnoid membrane. Moreover, if there are multiple tear points in the arachnoid membrane, the packing method is insufficient to prevent CSF leakage. For the prevention of these complications, we applied the snare technique for the remodeling of the redundant arachnoid pouch.

Before applying the snare technique, the positional relationship between the redundant arachnoid membrane and the normal gland should be investigated in the microscopic view. When the normal gland is located in the corner of the evaginated pouch and the arachnoid membrane comprises most of the evaginated pouch, the snare technique is preferred.

The snare was prepared on the table outside the surgical field using a 7-0 Pronova suture (Ethicon Inc.) with a short double-arm needle (7.6-mm taper) and specially designed suture-tying forceps, which were described in our previous report. ${ }^{2}$ An appropriately sized loop was created with a suture thread by making a double knot (Fig. 2A and $\mathrm{B}$ ), and both needles at the ends of the Pronova suture material passed from the tip-side holes on both jaws of the suture-tying forceps to the holes on the rear side (Fig. $2 \mathrm{C})$. The knot was grasped firmly using the suture-tying forceps to maintain the diameter of the loop (Fig. 2D). Subsequently, the suture-tying forceps holding the loop were introduced into the surgical field through a nasal speculum. The prolapsed redundant arachnoid membrane was grasped with grasping forceps and gently pulled out through the loop (Fig. 2E and F). The amount of arachnoid membrane held by the snare loop should be sufficient to prevent the formation of a voluminous dead space and to cover all the tear points of the arachnoid membrane for sealing off the CSF leakage fundamentally. At the same time, the surgeon should be aware that the snare loop should not include the normal gland to prevent its dysfunction. After confirmation of the proper positioning of the loop, the two threads of the suture material were pulled with moderate tension by a first surgical assistant, while the surgeon tightened the knot to constrict the loop via opening and closing maneuvers of the jaws of the suturetying forceps (Fig. 2G). After the constriction of the loop in the proper position, second and third knots were made to secure the first knot. During this procedure, the knots were carefully made outside the nasal speculum with mild tension on the 2 threads of the suture, and were slipped along the thread onto the first knot using suture-tying forceps. The knots were pushed and tied firmly to tighten the snare loop securely. Finally, the threads were cut with microscissors (Fig. 2H, Video 1).

VIDEO 1. Clip showing the snare technique in a step-by-step fashion. Copyright Sun Ho Kim. Published with permission. Click here to view.

\section{Sellar Floor Reconstruction}

Sellar floor reconstruction was performed using our routine technique, as described previously. ${ }^{8}$ After arachnoid repair using the snare technique, additional TachoSil-covered microfibrillar collagen hemostat (Avitene, MedChem Products Inc.) was placed over the arachnoid membrane for the purpose of hemostasis and for supporting the arachnoid membrane. Sellar floor reconstruction to cover the entire bone defect was performed using two kinds of materials: TachoSil was used in 7 cases and a porous polyethylene implant (Medpor, Porex Surgical Inc.) was used in 2 cases. After confirming that there was no CSF leakage, a topical tissue sealant (BioGlue, CryoLife 
A
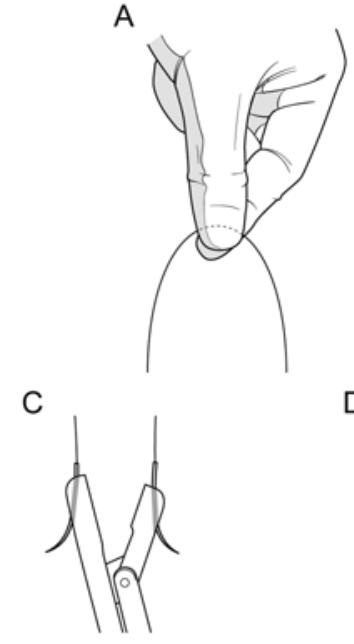

$\mathrm{E}$

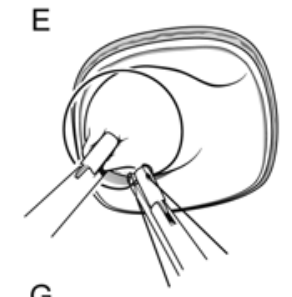

G

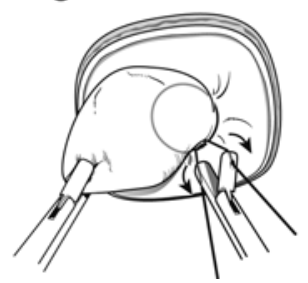

D
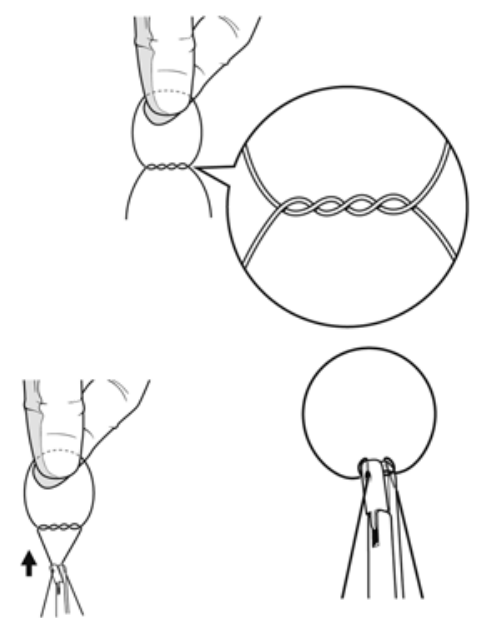

$\mathrm{F}$

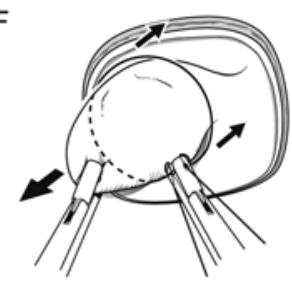

$\mathrm{H}$

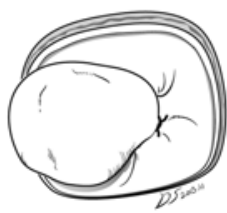

FIG. 2. Schematic description of the snare technique. A and B: A loop was created with a suture thread by making a double knot. C: Both needles at the ends of the Pronova suture material passed through both jaws of the suture-tying forceps. D: The knot was grasped firmly using the suture-tying forceps to maintain the diameter of the loop. E and F: The prolapsed redundant arachnoid membrane was gently pulled out through the loop. G: After confirmation of the proper positioning of the loop, the surgeon tightened the knot to constrict the loop via opening and closing maneuvers. H: Finally, the threads were cut with microscissors. Copyright Sun Ho Kim. Published with permission.

Inc.) was applied to cover the entire bone defect of the sellar floor, for the reinforcement of the sealing, and for prevention of the migration of reconstruction materials.

\section{Results}

Adventitious arachnoid tears occurred in 8 patients during the resection of the tumor. A single hole was identified in 3 patients, and multiple holes were observed in 5 patients. In 1 case in which CSF leakage did not develop, as the arachnoid pouch was intact, the constriction of the loop was not possible because of the large volume of CSF inside the redundant arachnoid pouch. There was a possibility of postoperative hematoma collection between the packing material and the redundant arachnoid membrane. Thus, we intentionally punctured the arachnoid and reduced the CSF volume in the pouch to facilitate the con-

striction of the snare loop, and then the snare loop was successfully tightened.

After tying the prolapsed arachnoid membrane using the snare technique, complete sealing of intraoperative CSF leakage was confirmed under the microscopic view in all cases. Postoperatively, CSF rhinorrhea developed in none of the 9 patients. Lumbar CSF drainage was not required in any of the patients. There were no other complications, including meningitis.

On the preoperative MR images, all tumors extended into the suprasellar space with compression of the optic apparatus. The height of the suprasellar extension from the diaphragm level ranged from 18 to $44.3 \mathrm{~mm}$, with an average height of $23.6 \mathrm{~mm}$. The early postoperative MR images taken 24 hours after surgery revealed a remarkable reduction of the height of the suprasellar extension in all cases. The height of the suprasellar extension from the diaphragm level on the early postoperative MR images ranged from 5.5 to $14.5 \mathrm{~mm}$, with an average height of $10.8 \mathrm{~mm}$. The average reduction of the height of the suprasellar extension was $51.9 \%$. MR images obtained 1 year after surgery revealed that the suprasellar extension had decreased remarkably because of the complete absorption of the implanted materials. The highest point of the contents of the sella turcica was below the diaphragm level in every case (Fig. 3A and B), with the exception of 2 cases in which the 1-year follow-up MRI study had not been performed.

Preoperatively, visual impairment was present in every patient because of the compression of the optic apparatus, as shown on the preoperative MR images. A classical bitemporal hemianopsia was noted in 6 patients and variations of the field defect pattern were noted in the other 3 patients. After resection of the tumors, the visual deficits improved in all patients.

\section{Discussion}

CSF leakage is a major postoperative complication of TSS that can cause life-threatening meningitis or pneumocephalus. The possibility of arachnoid tearing is clearly higher in macroadenomas with huge suprasellar extension, because the arachnoid membrane can be easily damaged during the dissection of the suprasellar tumor. The more effort that is made for complete and aggressive resection of tumors, the more likely it is that intraoperative CSF leakage with an arachnoid tear can be encountered. Despite the increased risk of CSF leakage, this effort for total resection of tumors, including removal of a microsurgical pseudocapsule, is crucial for the cure of the disease. Previously, we reported the importance of the removal of the pseudocapsule, which frequently contains tumor cells. ${ }^{25}$

If intraoperative CSF leakage occurs during TSS, the best method of preventing CSF rhinorrhea is complete repair of the leak point by sealing the arachnoid tear point during the initial TSS. However, in cases with multiple tears of the arachnoid membrane or a vague CSF leak point, this approach is not always applicable. The conventional packing technique that uses pieces of fat or muscle has commonly been used for the prevention of postoperative CSF leakage, with or without lumbar drainage. How- 
A Preop

Postop

24 hours

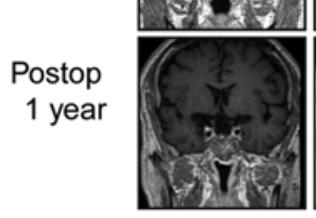

Case 1

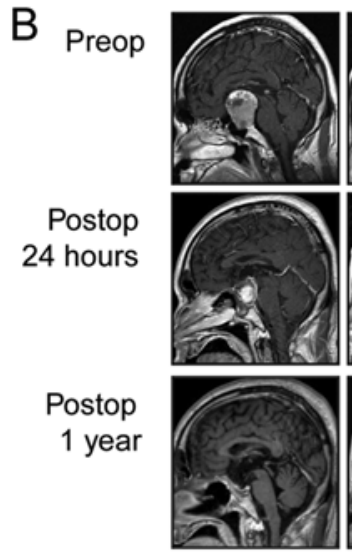

Case 1

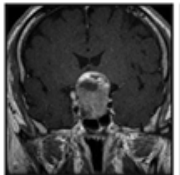

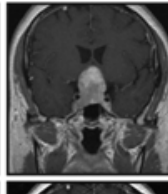
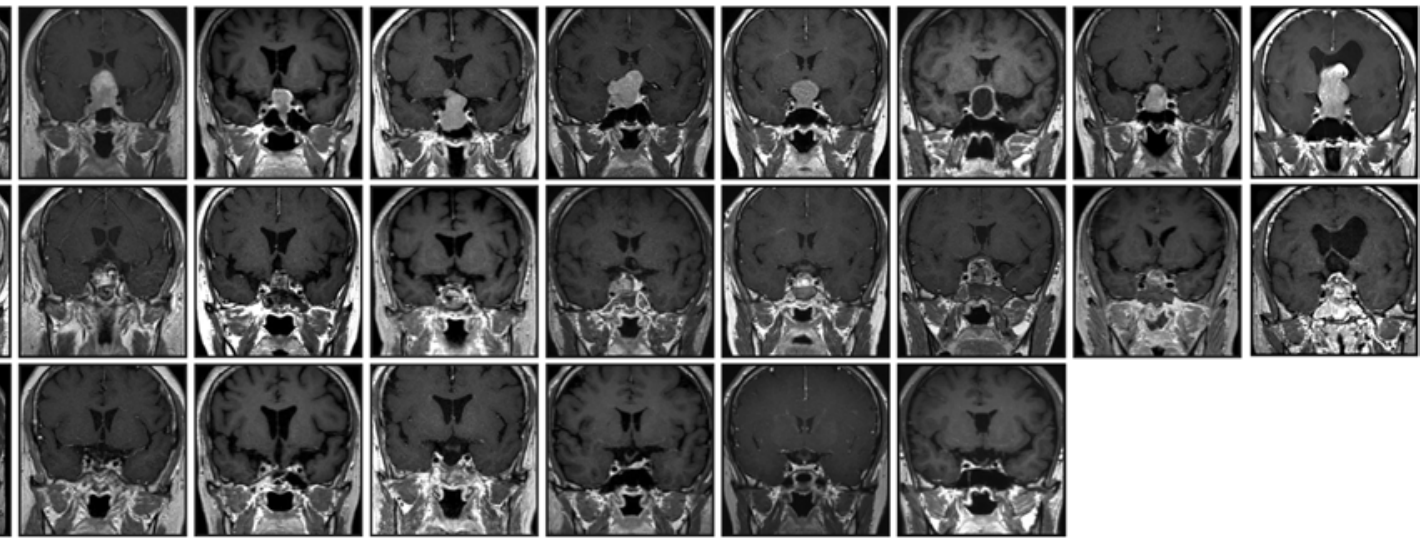

Case 2

Case 3
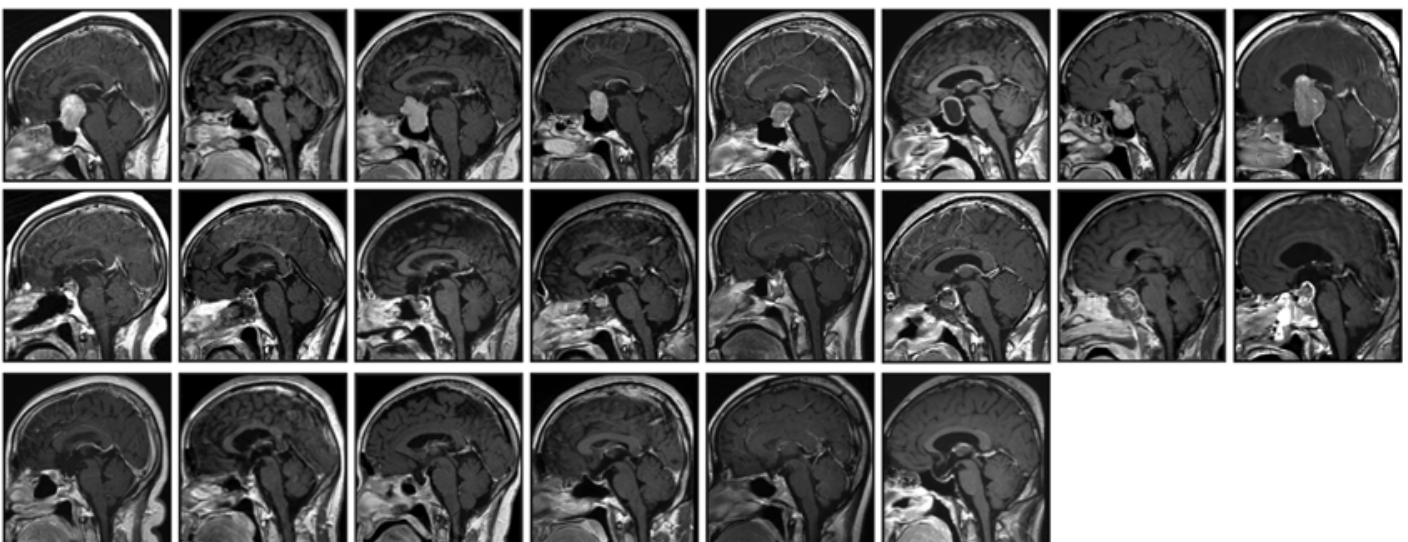

Case 2

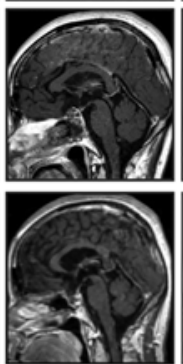

Case 3

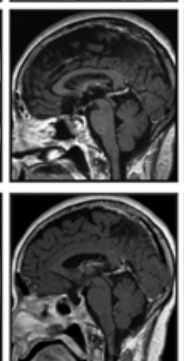

Case 4

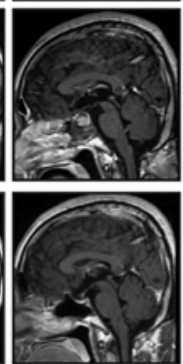

Case 5

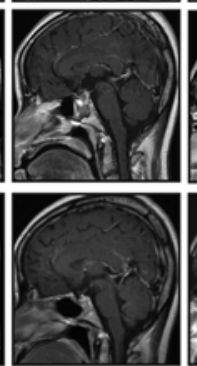

Case 6
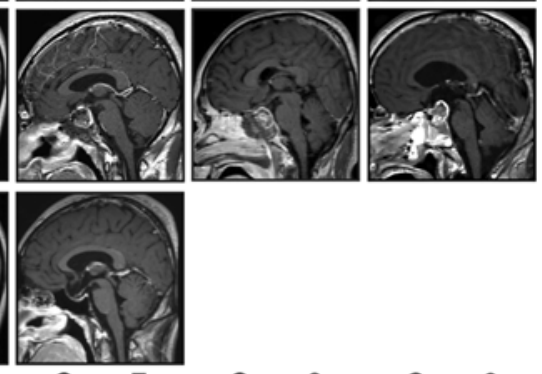

FIG. 3. Preoperative, early postoperative (24 hours), and 1-year coronal (A) and sagittal (B) MR images of the 9 patients in the study. After resection of a huge pituitary macroadenoma with suprasellar extension, the height of the diaphragm decreased remarkably in all patients.

ever, this technique may not be sufficient to seal the leak point completely, as the packing material cannot put firm pressure on the overlying arachnoid membrane because of the redundancy of the arachnoid membrane in the suprasellar space. Moreover, overpacking occasionally leads to compression of the surrounding neurovascular structures, and to consequent neurological symptoms.

Postoperative hematoma in the tumor resection cavity is occasionally observed after TSS for macroadenoma with suprasellar extension. Several studies reported that the early postoperative MR images showed that the persistent sellar contents consisted of accumulated postoperative hemorrhage, fluid collection, and hemostatic packing materials in almost all cases of macroadenomas with suprasellar extensions. ${ }^{12,17,22,30,31}$ Especially in cases with huge suprasellar extensions, postoperative hematoma can be accumulated in a large dead space of the suprasellar area after the removal of the tumor. Although the size of the postoperative sellar contents is usually similar or minimally decreased compared with that observed in preoperative MR images, it can increase with or without aggravation of neurological symptoms in some cases. Sometimes the increase in the size of the hematoma is fatal because it compresses adjacent neurovascu- lar structures. If a large postoperative hematoma filling the tumor resection cavity causes serious complications, such as visual disturbance, altered consciousness, or hypothalamic injury, it must be removed immediately by reoperation. Because reoperation is more dangerous than the first surgery, and neurological sequelae can remain despite the early removal of the postoperative hematoma, this complication should be avoided at all costs. However, the conventional packing method that uses pieces of fat or muscle may not be sufficient to prevent postoperative hematoma in the dead space.

To prevent postoperative CSF leakage and hematoma in the tumor resection cavity, we adopted purse-string suturing of the evaginated arachnoid membrane before the development of the snare technique. Similar to the snare technique, purse-string suturing can provide sealing of the CSF leakage and reduction of the postoperative tumor resection cavity by remodeling the prolapsed redundant arachnoid membrane. However, a complicated maneuver is required to introduce several stitches on the redundant arachnoid membrane in a narrow and deep surgical field; consequently, the operation time is prolonged. In cases with a weak and thin arachnoid membrane, its manipulation can induce the aggravation of arachnoid tears, and the 
various needle holes made by purse-string suturing may become additional CSF leak points.

The snare technique compensates for the disadvantages of the purse-string suturing technique. In the snare technique, the surgical maneuver is much easier than that used for purse-string suturing; thus, it is a shorter procedure, because the snare loop and additional knots are performed outside of the nasal speculum. In addition, because it tightens the large area of the arachnoid membrane at once, it is very effective in the repair of CSF leakage from multiple tear points on the arachnoid membrane. In our series, postoperative CSF rhinorrhea did not occur in any patient without the aid of postoperative lumbar drainage in any of the cases. Moreover, the height of the suprasellar extension and the dimension of the sellar contents evidently decreased in all patients, as assessed using postoperative MR images. These results imply that the snare technique is very effective for the complete sealing of CSF leakage and reduction of the tumor resection cavity.

Despite the simplicity and effectiveness of the snare technique, this technique could be useful in only limited cases. In our series, we have used the snare technique in only about $1 \%$ of our cases, and in less than $2 \%$ of our cases with suprasellar extension of tumor. It is possible to apply this technique exclusively for the cases with large redundant arachnoid membranes that herniate downward near or below the level of the dural opening after resection of tumor masses. In cases with small pituitary tumors that are localized in the sellae or extend slightly into the suprasellar space, the arachnoid membranes are not expanded enough to be manipulated for the snare technique. The condition of a redundant arachnoid membrane is another limiting factor for the application of the snare technique. If the redundant arachnoid membrane is too thin, meticulous care is required in manipulation of the arachnoid membrane because of the risk of disruption. When there is a large tear on the arachnoid or an adhesion between the arachnoid and structures located in the suprasellar space, the application of the snare technique would be impossible because the arachnoid membrane could not herniate downward but retracts upwards. Additionally, the neurovascular structures adjacent to the arachnoid membrane occasionally hinder the application of the technique. If a relatively thick layer of the normal pituitary gland covers the center of the arachnoid membrane, it could be injured by this technique, which may lead to worsening of hormonal function. In cases with a diffusely situated normal gland on the entire evaginated arachnoid membrane, a very small part of the exposed arachnoid membrane is available for tying a snare loop; consequently, the snare technique is not a good method in these cases. Arachnoid vessels are another nuisance, because hemorrhage in the subarachnoid space and infarction of the surrounding structures that are fed by the vessels may be provoked when the vessels are located inside the loop and are tied. If vessels are identified on the arachnoid membrane, they should always remain outside the tying portion of the redundant arachnoid membrane. Moreover, extra attention is required to avoid damage to the subarachnoid neurovascular structures, including the optic apparatus, the pituitary stalk, and the perforating arteries. A large volume of CSF inside the arachnoid pouch can also interfere with the constriction of the snare loop. As described earlier, intentional puncturing of the arachnoid membrane can reduce the volume and tension of the arachnoid pouch by draining CSF. Before constricting and tying the loop, surgeons should check whether the loop is properly placed so that the puncture site is located inside it.

During the period of this study, there were 158 cases of pituitary tumors with huge suprasellar extension in which the arachnoid membranes were redundantly herniated downward after the resection of the tumor. Except for the 9 cases of this series in which the arachnoid membranes were treated with the snare technique, the arachnoid membranes were treated with several other techniques in the rest of the 149 cases. A lump of TachoSil covering a small piece of Avitene was directly applied in 73 cases, mostly for the cases without a definite arachnoid tear. ${ }^{8}$ The coaptation method $^{8}$ was applied in 20 cases with a small single leakage point, while the evaginated arachnoid membrane was coagulated and shrunk the herniated arachnoid sac. In 39 cases with a diffusely situated normal gland on the entire evaginated arachnoid membrane, we used the direct suture technique of normal gland edge on the incised dura margin for the reconstruction of the diaphragm. ${ }^{20}$ In 6 cases, the coagulation and direct suture techniques were applied together. The purse-string suturing technique was applied in 4 cases with small exposure of prolapsed arachnoid membrane. When the exposure of prolapsed arachnoid membrane is small because of the centrally located normal pituitary gland, or when an arachnoid membrane is partially evaginated because of adhesion between the arachnoid membrane and suprasellar structures, it is technically difficult to place the loop in the proper position and tie it. In this situation, the purse-string suturing technique could be advantageous for tying the arachnoid membrane, as the placement of direct stitches on the arachnoid membrane secures the position of the suture over it. Six cases with large arachnoid defects were treated with attachment of a small piece of free septal mucosal flap. In 1 case, we used the conventional packing method using fat and fascia lata. Unfortunately, among these 149 cases, 2 experienced postoperative CSF rhinorrhea and required operations for repair of CSF leakage, and 1 case required an immediate reoperation for evacuation of a postoperative hematoma.

In the early phase of application of the snare technique, we failed to apply this technique in 3 cases because we did not have enough experiences to handle very thin arachnoid membranes. The redundant arachnoid membranes were relatively thin in these cases, so they tore while the snare loops were being tightened. Despite the relatively little manipulation of this technique, special caution is required during the manipulation of redundant arachnoid membrane, because there is always a risk of disruption of thinned arachnoid membrane. The disrupted arachnoid membranes were repaired with direct suture of the torn arachnoid in 2 cases and with purse-string suturing in another case, and fortunately no symptom related to the postoperative CSF leakage and hematoma developed in these cases.

\section{Conclusions}

The snare technique is very useful for the reconstruc- 
tion of the redundant evaginated arachnoid membrane after the resection of huge macroadenomas in selected cases. Despite the limited applicability of the technique, the technical simplicity and the encouraging clinical outcomes reported by our study demonstrate the efficacy of the snare technique in preventing postoperative CSF rhinorrhea and hematoma formation in the tumor resection cavity.

\section{Acknowledgments}

We thank Mr. Dong Su Jang, a medical illustrator, for his beautiful work on the figures in this paper.

\section{References}

1. Adams CB: The management of pituitary tumours and post-operative visual deterioration. Acta Neurochir (Wien) 94:103-116, 1988

2. Ahn JY, Kim SH: A new technique for dural suturing with fascia graft for cerebrospinal fluid leakage in transsphenoidal surgery. Neurosurgery 65 (6 Suppl):65-72, 2009

3. Barker FG II, Klibanski A, Swearingen B: Transsphenoidal surgery for pituitary tumors in the United States, 1996-2000: mortality, morbidity, and the effects of hospital and surgeon volume. J Clin Endocrinol Metab 88:4709-4719, 2003

4. Barrow DL, Tindall GT: Loss of vision after transsphenoidal surgery. Neurosurgery 27:60-68, 1990

5. Barzaghi LR, Losa M, Giovanelli M, Mortini P: Complications of transsphenoidal surgery in patients with pituitary adenoma: experience at a single centre. Acta Neurochir (Wien) 149:877-886, 2007

6. Black PM, Zervas NT, Candia G: Management of large pituitary adenomas by transsphenoidal surgery. Surg Neurol 29:443-447, 1988

7. Black PM, Zervas NT, Candia GL: Incidence and management of complications of transsphenoidal operation for pituitary adenomas. Neurosurgery 20:920-924, 1987

8. Cho JM, Ahn JY, Chang JH, Kim SH: Prevention of cerebrospinal fluid rhinorrhea after transsphenoidal surgery by collagen fleece coated with fibrin sealant without autologous tissue graft or postoperative lumbar drainage. Neurosurgery 68 (1 Suppl Operative):130-137, 2011

9. Ciric I, Ragin A, Baumgartner C, Pierce D: Complications of transsphenoidal surgery: results of a national survey, review of the literature, and personal experience. Neurosurgery 40:225-237, 1997

10. Couldwell WT: Transsphenoidal and transcranial surgery for pituitary adenomas. J Neurooncol 69:237-256, 2004

11. Decker RE, Chalif DJ: Progressive coma after the transsphenoidal decompression of a pituitary adenoma with marked suprasellar extension: report of two cases. Neurosurgery 28:154-158, 1991

12. Dina TS, Feaster SH, Laws ER Jr, Davis DO: MR of the pituitary gland postsurgery: serial MR studies following transsphenoidal resection. AJNR Am J Neuroradiol 14:763-769, 1993

13. Goel A, Nadkarni T: Surgical management of giant pituitary tumours-a review of 30 cases. Acta Neurochir (Wien) 138:1042-1049, 1996

14. Goel A, Nadkarni T, Muzumdar D, Desai K, Phalke U, Sharma P: Giant pituitary tumors: a study based on surgical treatment of 118 cases. Surg Neurol 61:436-446, 2004

15. Guiot G, Derome P: Surgical problems of pituitary adenomas, in Krayenbühl H, Brihaye J, Loew F, et al (eds): Advances and Technical Standards in Neurosurgery. Vienna: Springer, 1976, pp 3-33

16. Jane JA Jr, Thapar K, Kaptain GJ, Maartens N, Laws ER Jr: Pituitary surgery: transsphenoidal approach. Neurosurgery 51:435-444, 2002
17. Kiliç T, Ekinci G, Seker A, Elmaci I, Erzen C, Pamir MN: Determining optimal MRI follow-up after transsphenoidal surgery for pituitary adenoma: scan at 24 hours postsurgery provides reliable information. Acta Neurochir (Wien) 143:1103-1126, 2001

18. Kim EH, Ku CR, Lee EJ, Kim SH: Extracapsular en bloc resection in pituitary adenoma surgery. Pituitary 18:397-404, 2015

19. Kim EH, Oh MC, Kim SH: Application of low-field intraoperative magnetic resonance imaging in transsphenoidal surgery for pituitary adenomas: technical points to improve the visibility of the tumor resection margin. Acta Neurochir (Wien) 155:485-493, 2013

20. Kim EH, Roh TH, Park HH, Moon JH, Hong JB, Kim SH: Direct suture technique of normal gland edge on the incised dura margin to repair the intraoperative cerebrospinal fluid leakage from the arachnoid recess during transsphenoidal pituitary tumor surgery. Neurosurgery 11 (2 Suppl 2):26-31, 2015

21. Kitano M, Taneda M: Capsule plication as a protective measure against post-operative intracapsular haematoma formation following trans-sphenoidal removal of pituitary macroadenoma. Acta Neurochir (Wien) 150:797-802, 2008

22. Kremer P, Forsting M, Ranaei G, Wüster C, Hamer J, Sartor $\mathrm{K}$, et al: Magnetic resonance imaging after transsphenoidal surgery of clinically non-functional pituitary macroadenomas and its impact on detecting residual adenoma. Acta Neurochir (Wien) 144:433-443, 2002

23. Ku CR, Kim EH, Oh MC, Lee EJ, Kim SH: Surgical and endocrinological outcomes in the treatment of growth hormone-secreting pituitary adenomas according to the shift of surgical paradigm. Neurosurgery 71 (2 Suppl Operative):ons192-ons203, 2012

24. Kurwale NS, Ahmad F, Suri A, Kale SS, Sharma BS, Mahapatra AK, et al: Post operative pituitary apoplexy: preoperative considerations toward preventing nightmare. Br J Neurosurg 26:59-63, 2012

25. Lee EJ, Ahn JY, Noh T, Kim SH, Kim TS, Kim SH: Tumor tissue identification in the pseudocapsule of pituitary adenoma: should the pseudocapsule be removed for total resection of pituitary adenoma? Neurosurgery 64 (3 Suppl):ons62ons70, 2009

26. Liu JK, Das K, Weiss MH, Laws ER Jr, Couldwell WT: The history and evolution of transsphenoidal surgery. J Neurosurg 95:1083-1096, 2001

27. Matsuyama J, Kawase T, Yoshida K, Hasegawa M, Hirose Y, Nagahisa S, et al: Management of large and giant pituitary adenomas with suprasellar extensions. Asian J Neurosurg 5:48-53, 2010

28. Nakane T, Kuwayama A, Watanabe M, Kageyama N: Transsphenoidal approach to pituitary adenomas with suprasellar extension. Surg Neurol 16:225-229, 1981

29. Park HJ, Lim JW, Lim YH, Rho YS: [A case of blindness after transsphenoid approach for pituitary adenoma removal: revision treatment experience under local anesthesia.] J Rhinol 12:120-123, 2005 (Korean)

30. Rajaraman V, Schulder M: Postoperative MRI appearance after transsphenoidal pituitary tumor resection. Surg Neurol 52:592-599, 1999

31. Rodríguez O, Mateos B, de la Pedraja R, Villoria R, Hernando JI, Pastor A, et al: Postoperative follow-up of pituitary adenomas after trans-sphenoidal resection: MRI and clinical correlation. Neuroradiology 38:747-754, 1996

32. Shou XF, Li SQ, Wang YF, Zhao Y, Jia PF, Zhou LF: Treatment of pituitary adenomas with a transsphenoidal approach. Neurosurgery 56:249-256, 2005

33. Wilson CB, Dempsey LC: Transsphenoidal microsurgical removal of 250 pituitary adenomas. J Neurosurg 48:13-22, 1978 
34. Zhang X, Fei Z, Zhang J, Fu L, Zhang Z, Liu W, et al: Management of nonfunctioning pituitary adenomas with suprasellar extensions by transsphenoidal microsurgery. Surg Neurol 52:380-385, 1999

\section{Disclosures}

The authors report no conflict of interest concerning the materials or methods used in this study or the findings specified in this paper.

\section{Author Contributions}

Conception and design: SH Kim. Acquisition of data: Moon. Analysis and interpretation of data: Moon. Drafting the arti- cle: Moon. Critically revising the article: SH Kim, EH Kim. Reviewed submitted version of manuscript: all authors. Approved the final version of the manuscript on behalf of all authors: $\mathrm{SH}$ Kim. Administrative/technical/material support: SH Kim, EH Kim. Study supervision: SH Kim.

\section{Supplemental Information}

Videos

Video 1. https://vimeo.com/149287035.

\section{Correspondence}

Sun Ho Kim, Department of Neurosurgery, Yonsei University College of Medicine, 50 Yonsei-ro, Seodaemun-gu, Seoul, 120752, Republic of Korea. email: sunkim@yuhs.ac. 